\title{
The chiral condensate on two-flavor staggered configurations from an overlap operator
}

\author{
Anna Hasenfratz* and Roland Hoffmann \\ Department of Physics, University of Colorado, Boulder, CO-80309-390, USA \\ E-mail: anna@eotvos.colorado.edu
}

\begin{abstract}
We measure the low lying eigenmodes of an overlap Dirac operator on 2-flavor staggered configurations. By comparing the eigenmode distribution to the predictions of Random Matrix Theory we test to what accuracy staggered configurations describe continuum QCD. The agreement between the numerical data and RMT implies that at our quark mass values the lattice artifacts of the staggered configurations are comparable to overlap configurations. We identify the overlap valence mass that best matches the staggered sea quarks and predict the value of the infinite volume 2-flavor chiral condensate.
\end{abstract}

XXIVth International Symposium on Lattice Field Theory

July 23-28, 2006

Tucson, Arizona, USA

\footnotetext{
* Speaker.
} 


\section{Introduction}

Mixed action simulations became popular in recent years as they combine the simulation advantages of a simple sea quark action with the exact or near exact chiral symmetry of overlap or domain wall valence quarks. The price to pay, in addition to an internal inconsistency (unitarity violation), is the complication in the analysis. One option is to derive and use partially quenched mixed action chiral perturbative formulae. Alternatively, one can match the parameters of the valence and sea quark actions as well as possible and deal with any remaining difference as part of the lattice artifacts. This approach is useful if the chiral perturbative formulae do not exist or the numerical data does not allow the fitting of all the parameters, or if one desires more insight into the physics contained in a particular set of gauge configurations.

The effectiveness of the latter approach was illustrated in Refs. [1, 2], where we showed that, at least within the 2-dimensional Schwinger model, mixed action simulations with overlap valence quarks on rooted or unrooted staggered sea quark configurations reproduce the full dynamical overlap results if the overlap mass is tuned appropriately. In this work we report our first results along the same lines in 4-dimensional 2-flavor QCD. We show that configurations generated with 2-flavor staggered quarks at a single lattice spacing but at four different quark masses are consistent with 2-flavor QCD configurations, at least for the three heavier masses. We also show how the topological charge distributions can be used to determine the best overlap valence matching masses and that with these mass values the data sets predict a consistent value for the chiral condensate of 2-flavor QCD. All details can be found in Ref. [3].

\section{Strategy and Simulation setup}

Our sea quark action is the 2-flavor Asqtad staggered action [4-6]. We have generated four configurations sets, each consisting of 400-500 $12^{4}$ lattices at a lattice spacing of about $a=0.13 \mathrm{fm}$. The details of the sets are summarized in Table 1. The level of taste breaking, the ratios of the heaviest and lightest pion masses, is approximated from corresponding $2+1$ flavor results [5]. The last column lists the separation of the configurations in terms of unit length molecular dynamics trajectories.

Our valence action is an overlap action based on an improved Wilson kernel on HYP smeared links. This action was used in recent overlap simulations [7, 8].

To investigate if the rooted staggered gauge ensembles are consistent with two-flavor continuum QCD one has to consider observables that are sensitive to the vacuum and do not depend strongly on the valence quark mass. Spectral quantities are not appropriate, but the low lying infrared eigenmodes of the massless valence Dirac operator offer a good choice. Another quantity which we will consider is the topological charge of the configurations. On each configurations we ask what valence quark mass matches the staggered configurations the best, i.e. what valence mass minimizes the lattice artifacts, the difference between lattice data and continuum QCD.

\section{Eigenvalues of the Dirac Operator and Random Matrix Theory}

Random matrix theory (RMT) captures the universal chiral properties of QCD and predicts the distribution of the physical (infrared) eigenvalues of the massless Dirac operator in the $\varepsilon$-regime. 


\begin{tabular}{|c|c|c|c|c|c|}
\hline Set & $\beta$ & $a m_{s t}$ & $r_{0} / a[9,10]$ & Taste breaking & Time separation \\
\hline \hline $\mathbf{L}$ & 7.18 & 0.01 & $3.84(6)$ & $60 \%$ & 5 \\
\hline $\mathbf{M}$ & 7.20 & 0.02 & $3.82(3)$ & $34 \%$ & 5 \\
\hline $\mathbf{H}$ & 7.22 & 0.03 & $3.60(4)$ & $24 \%$ & 10 \\
\hline $\mathbf{E}$ & 7.24 & 0.04 & $3.64(3)$ & $18 \%$ & 15 \\
\hline
\end{tabular}

Table 1: Parameters of the $n_{f}=2$ staggered background configurations. The molecular dynamics time separations between the configurations reflect the autocorrelation of the topological charge.

The predictions are given in fixed topological sector $v$ and depend on the low energy constant $\Sigma$, the infinite volume chiral condensate. The distribution of the (microscopically rescaled) $n^{\text {th }}$ eigenmode $\lambda \Sigma V$ is given as

$$
P_{V, n}(\lambda \Sigma V)=\Lambda_{v, n}\left(m \Sigma V ; n_{f}\right),
$$

where $m$ is the quark mass of the configurations (sea quark mass), which in our case is an overlap quark mass that corresponds to the background configurations that were generated by staggered quarks, i.e. $m$ is the matching quark mass as described in Sect. 2. The value of $m$ is not known a priori and therefore $\Lambda_{V, n}$ depends on two variables, $M=m \Sigma V$ and $\Sigma$. We fit the measured eigenvalue distribution to random matrix theory at fixed $M$ and predict the chiral condensate $\Sigma$. The systematic deviation of the data from the RMT prediction of Eq.(3.1) characterizes the lattice artifacts, both from discretization errors and from the non-locality of the action. This deviation is the measure of consistency between the lattice action and continuum QCD and replaces the residue used in Ref.[1] for the same purpose. If the rooting procedure is correct, it should scale to zero as the continuum limit is approached at fixed physical (matching) quark mass, assuming the simulations are done in the region where the RMT predictions are valid.

To fit the cumulative distributions, we use the Kolmogorov-Smirnov (KS) test that minimizes $D_{\max }^{2}$, the maximal deviation between the measured and the predicted cumulative distributions [1113]. An advantage of the KS test is that there is an explicit and simple form for the confidence level of the fit. For a given sample length this quality factor $Q_{\mathrm{KS}}$ is a monotonically decreasing function of $D_{\max }$ that gives the probability that the measured distribution is consistent with the analytical one. The KS fit maximizes the quality factor $Q_{\mathrm{KS}}$ or the product of quality factors if more than one distribution is used.

However, $Q_{\mathrm{Ks}}$ will go to zero exponentially with increasing statistics if the measured distribution is not exactly described by the analytic form. In any lattice calculations there are lattice artifacts and finite volume effects, so the analytic form is never exactly reproduced, the quality factor vanishes as the numerical statistics increases. In the following we fit our data by maximizing the quality factor (or products of quality factors) according to the KS test, but describe the goodness of the fit by the value $D_{\max }$ itself to enable a comparison with other results.

Fig. 1 shows a typical fit of the cumulative distribution for the $\mathbf{M}\left(a m_{s t}=0.02\right)$ data set at $M=13.5$. The left panel corresponds to the $v=0$, the right panel to the $v=1$ sector. Only the first modes of the $v=0$ and 1 topological sectors are included in the fit. In addition to the two fitted modes we also show the non-fitted second modes in the same topological sectors. $D_{\max }$ is almost a factor of two smaller for the $n=1, v=1$ mode, but not significantly worse for the non-fitted modes than for the fitted $n=1, v=0$ mode. 

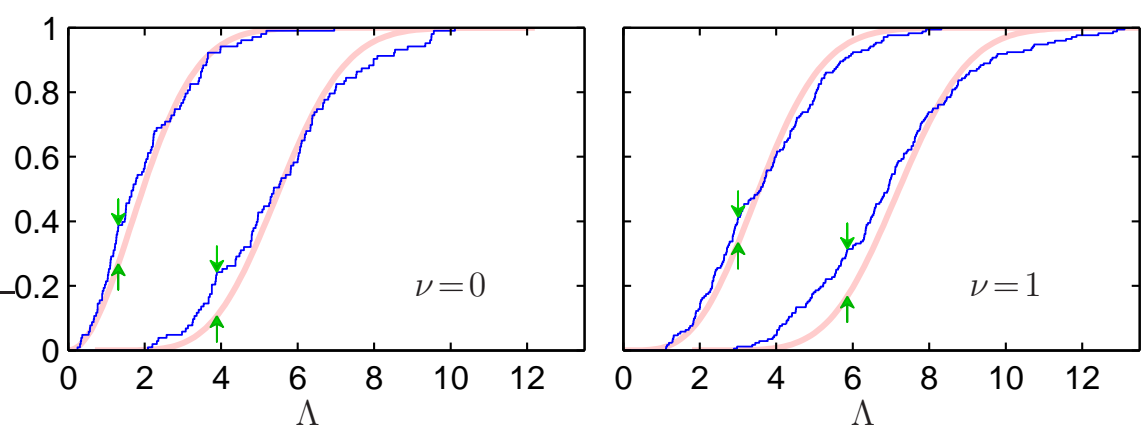

Figure 1: RMT predictions of the cumulative distribution of the two lowest eigenmodes in the $v=0$ and 1 sectors of the $\mathbf{M}$ set at $M=13.5$ (see below). The fit uses only the first mode in each topological sector. Arrows indicate the maximal deviation between the data set and the analytical predictions.

In Fig.2 we plot the maximal deviations $D_{\max }$ as a function of the RMT parameter $M$. Evidently the quality of the fit is not very sensitive to the parameter $M$. While small values are disfavored, larger values are almost equally probable. Contrary to our original hopes the eigenmode distributions cannot be used to define a matching mass, it defines only a range of acceptable values.

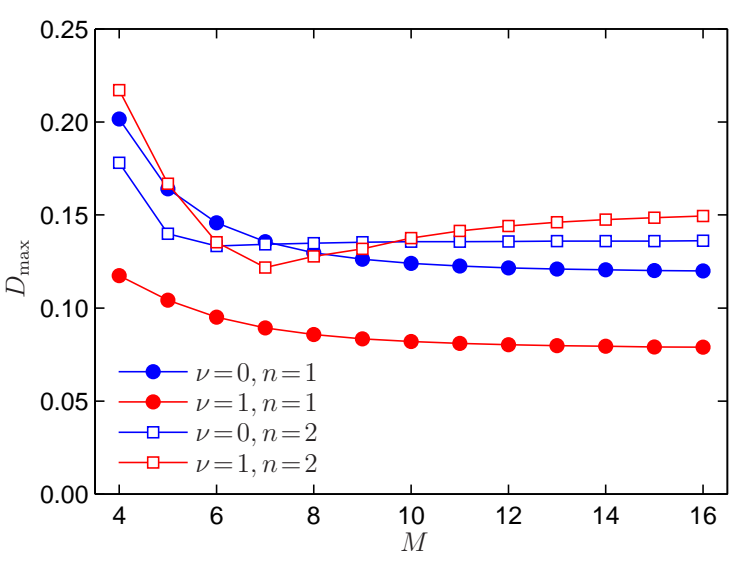

Figure 2: $D_{\max }$ as the function of $M$ for the $\mathbf{M}$ data set. The fit uses the first modes of the $v=0$ and 1 sectors (filled points).
Result of the fit are similar for the other three data sets. The upper panels of Figure 3 show $D_{\max }$ for the fitted modes, and the dependence on the staggered mass is obvious. $D_{\max }$ is significantly lower at the heaviest $\mathbf{E}$ data set than for the lightest $\mathbf{L}$ one, with the intermediate mass sets lying in between. This behavior is expected since at finite lattice spacing a smaller staggered mass leads to increased taste symmetry breaking (Table 1), it differs more from the flavor symmetric valence quark sector. With decreasing lattice spacing at fixed physical quark mass this deviation should decrease and eventually vanish in the continuum limit.

At each $M$ value the fit predicts $\Sigma V / a$ and using $r_{0} / a$ from Table 1 this can be converted to physical units as shown on the lower panels of Fig.3. The corresponding overlap mass values $m$ are shown along the upper border of the figure. In order to predict the chiral condensate we have to find an independent quantity that predicts the matching valence quark mass. The topological charge distribution is a possible choice as we will discuss in Sect. 4.

In Table 2 we list the number of configurations, the $D_{\max }$ values of the RMT fit at specific $M=m \Sigma V$ values and the corresponding quality factors. The $D_{\max }$ values can be compared to those from Ref. [13]. That work uses dynamical overlap configurations at similar physical volumes at slightly coarser lattice spacing. Using the same fitting strategy as ours they find $D_{\max }=0.11 \sim 0.20$. In view of these numbers we can conclude that, as far as the Dirac operator eigenmode distribution 


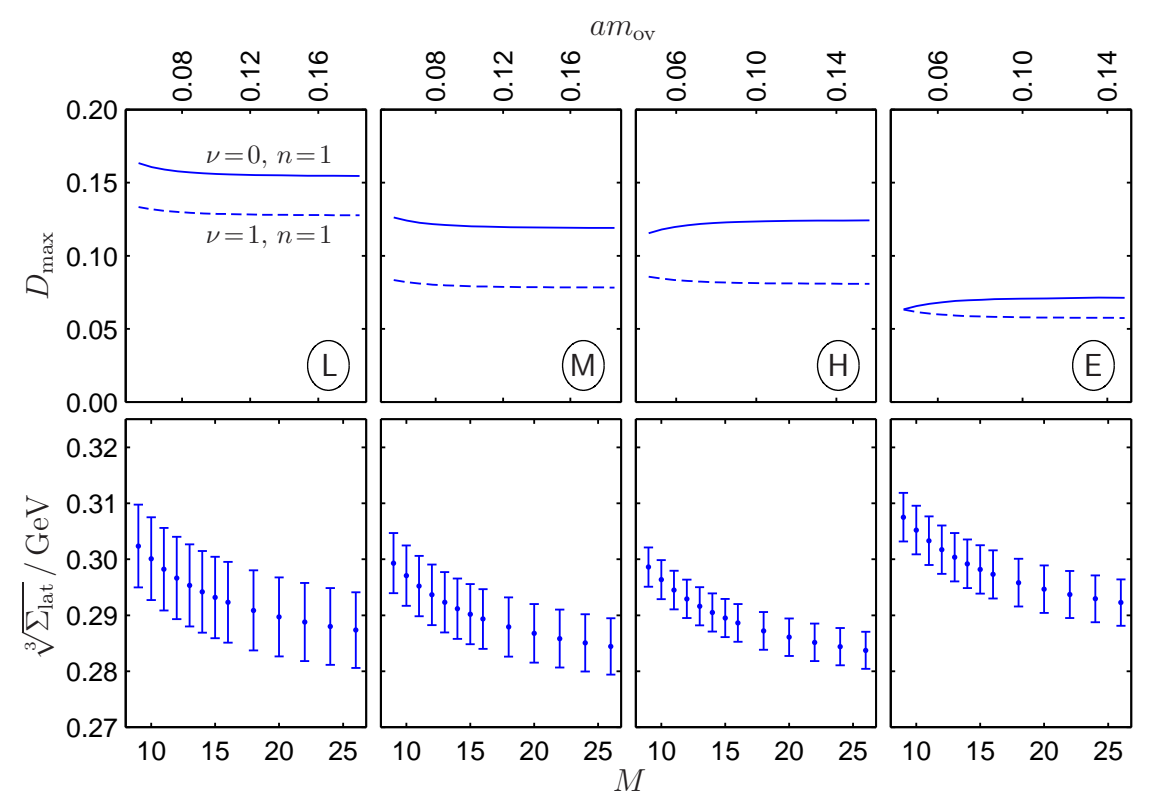

Figure 3: $D_{\max }$ and $\Sigma^{1 / 3}$ in $\mathrm{GeV}$ as the function of $M=m \Sigma V$ for all four data sets.

is concerned, the rooted staggered action configurations do not show larger lattice artifacts than the overlap ones.

\section{Topology}

Since we have sufficient statistics, over 400 approximately independent configurations at each coupling value on not too large volumes $\left(12^{4}\right.$ or about $\left.6 \mathrm{fm}^{4}\right)$, we can study the topological charge distribution. Following the discussion of Refs. [14-16], we write the probability of encountering a charge $\pm v$ configuration in the dynamical ensemble as $P_{v}=Z_{v}(m \Sigma V) Q_{v}(\sigma)$. Here $Q_{v}$ is the quenched probability of a charge $\pm v$ configuration, expected to be Gaussian up to $1 / V$ corrections, while $Z_{v}$ describes the suppression due to the fermionic determinant. The fermionic suppression factor has been calculated both within chiral perturbation theory and the random matrix model

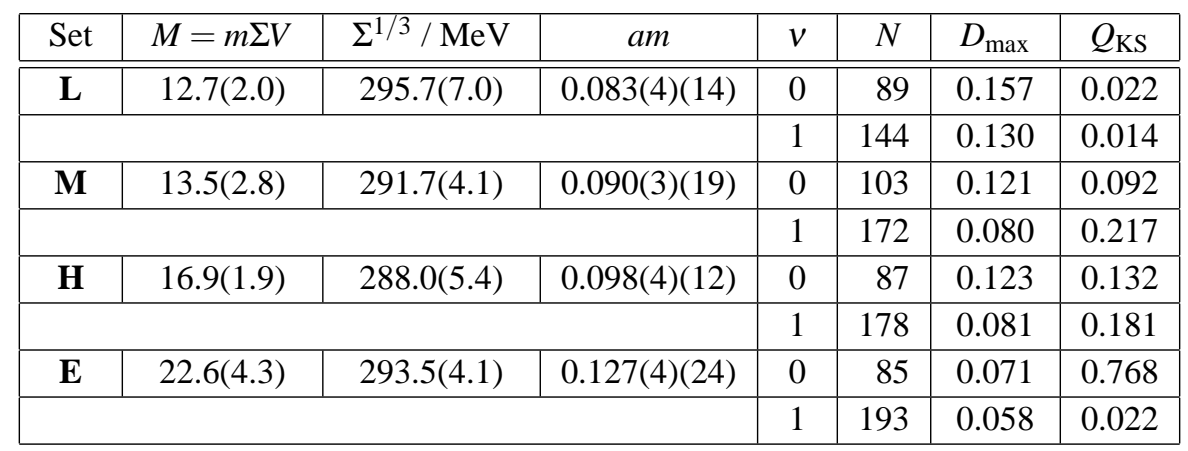

Table 2: Results of the RMT fit to the lowest eigenmodes in the $v=0,1$ sectors. For the determination of $M$ see Sect. 4. The first error on the matching overlap mass am is due to the uncertainty of $\Sigma$ only, while the second one takes into account both the errors of $\Sigma$ and $M$. 
$[14,15]$. Thus the charge probability distribution $P_{v}$ depends on two variables, $M=m \Sigma V$ and $\sigma$. The latter can be determined from the quenched topological susceptibility, so a one parameter fit to the topological charge distribution data predicts $M$, which we list in Table 2.

\section{The chiral condensate and matching masses}

With the $M=m \Sigma V$ values predicted from the topological charge distribution we are now able to extract the physical value of the chiral condensate. Combining the $M$ values with $r_{0} / a$ from Table 1 we find that all four configuration sets predict a consistent value for the $\Sigma$ condensate, as listed in Table 2. The only sign that the light $\mathbf{L}$ set differs from the RMT prediction more than the other mass values is the larger error of the predicted condensate. The value we obtain,

$$
\Sigma_{\text {lat }}^{1 / 3}=291(5) \mathrm{MeV},
$$

is the lattice condensate. It is consistent with predictions obtained on overlap dynamical configurations [13], further supporting our observation that the rooted staggered configurations are QCD like, the non-local terms of the action can be simply taken into account as lattice artifacts.

To connect the value of the condensate to a more conventional scheme, like $\overline{\mathrm{MS}}$ at $2 \mathrm{GeV}$, one needs the corresponding renormalization factor $Z_{s}$. Such a factor should be calculated nonperturbatively on the staggered configurations with our specific valence Dirac operator. We have not done this calculation yet but similar ones exist $[12,13,17] . Z_{s}$ seems to be largely independent of the detailed properties of the background configurations and we estimate its value to be $Z_{s} \geq 0.9$, which will lower $\Sigma_{\text {lat }}^{1 / 3}$ by $3 \%$. In addition, there is a finite volume correction to the condensate that could lower its value further [13]. These effects will have to be investigated but they are beyond the scope of the present work.

Combining $M$ and $\Sigma$ we get the values listed in the "am" column of Table 2 . These matching masses are not only surprisingly large but they do not depend linearly on the staggered masses. While the staggered quark mass changes a factor of four between the lightest and heaviest data sets, the matching overlap masses change only $50 \%$. This is similar to what we observed in the Schwinger model [1]. The matching valence masses show an overall shift compared to the staggered sea mass values. In addition at very small sea quark masses, where the matching breaks down, the valence quark masses are largely independent of the sea quark mass values. This is illustrated in Fig. 3 of Ref. [1]. Such behavior implies that staggered configurations at small quark masses are not necessarily closer to chiral continuum QCD than the heavier mass configurations. All the computational efforts creating light configurations might be in vain, creating only configurations with larger lattice artifacts. This might not be a problem when the data is analyzed with the whole machinery of staggered partially quenched chiral perturbation theory but should be considered when individual configuration sets are analyzed in mixed action simulations. Of course this is only a lattice artifact and any such effect will disappear as the continuum limit is approached.

\section{Conclusion}

We have studied the properties of the rooted staggered action in a mixed action simulation using overlap valence quarks. By comparing physical quantities that are independent of the valence quark mass to continuum QCD predictions we identified lattice artifacts and studied their 
dependence on the sea quark masses. In this work we considered the eigenvalue distribution of the massless Dirac operator and the distribution of the topological charge. We compared the former to the universal predictions of random matrix theory and found that the systematic deviation of the data from the predictions were comparable to dynamical overlap simulations. Using the topological charge distribution we could identify the matching overlap valence quark mass value which best describes the staggered configurations. We found these matching values to be fairly large and their dependence on the staggered sea mass values indicate a finite offset, in addition to a linear mass renormalization factor between the valence and sea mass values. With the use of this matching mass we extracted the value of the chiral scalar condensate. We found that the predictions from all of our staggered configuration sets were consistent. These findings indicate that at our lattice spacing, $a \approx 0.13 \mathrm{fm}$, and with not very light sea quarks the rooted staggered lattice configurations have lattice artifacts similar to other lattice actions, the non-local terms arising from the rooting procedure can be simply considered as part of the cutoff effects. In order to show that these nonlocal terms indeed become irrelevant in the continuum limit the calculation have to be repeated at different lattice spacings and the scaling of the lattice artifacts should be investigated. It would also be important to study in a similar manner the lattice artifacts of other observables.

\section{References}

[1] A. Hasenfratz and R. Hoffmann, Phys. Rev. D74 (2006) 014511, [hep-lat / 0604010 ].

[2] A. Hasenfratz and R. Hoffmann, PoS LAT2006 (2006) 212, [hep-lat/ 0609030 ].

[3] A. Hasenfratz and R. Hoffmann, Mixed action simulations on staggered background; interpretation and result for the 2-flavor QCD chiral condensate, hep-lat/0609067.

[4] MILC Collaboration, K. Orginos, D. Toussaint, and R. L. Sugar, Phys. Rev. D60 (1999) 054503, [hep-lat/9903032].

[5] C. W. Bernard et al., Phys. Rev. D64 (2001) 054506, [hep-lat/ 0104002 ].

[6] C. Aubin et al., Phys. Rev. D70 (2004) 094505, [hep-lat/ 0402030 ].

[7] MILC Collaboration, T. A. DeGrand, Phys. Rev. D63 (2001) 034503, [hep-lat / 0007046 ].

[8] MILC Collaboration, T. A. DeGrand, Phys. Rev. D69 (2004) 014504, [hep-lat/ 0309026 ].

[9] R. Sommer, Nucl. Phys. B411 (1994) 839-854, [hep-lat/9310022].

[10] A. Hasenfratz, R. Hoffmann, and F. Knechtli, Nucl. Phys. Proc. Suppl. 106 (2002) 418-420, [hep-lat/0110168].

[11] W. Bietenholz, K. Jansen, and S. Shcheredin, JHEP 07 (2003) 033, [hep-lat / 0306022 ].

[12] T. DeGrand, R. Hoffmann, S. Schaefer, and Z. Liu, Phys. Rev. D74 (2006) 054501, [hep-th/0605147].

[13] T. DeGrand, Z. Liu, and S. Schaefer, Quark condensate in two-flavor QCD, hep-lat/ 0608019.

[14] H. Leutwyler and A. Smilga, Phys. Rev. D46 (1992) 5607-5632.

[15] J. J. M. Verbaarschot and T. Wettig, Ann. Rev. Nucl. Part. Sci. 50 (2000) 343-410, [hep-ph/0003017].

[16] S. Dürr, Nucl. Phys. B611 (2001) 281-310, [hep-lat/0103011].

[17] T. A. DeGrand and Z. Liu, Phys. Rev. D72 (2005) 054508, [hep-lat/ 0507017$].$ 